\section{Oncology nurses' knowledge, practice, and confidence toward chemotherapy-induced peripheral neuropathy in Jordan}

\author{
Nijmeh M. Al-Atiyyat, RN, PhD, \\ Almutazbillah Z. Banifawaz, RN, MSN.
}

\section{ABSTRACT}

Objectives: To assess the knowledge, practice, and confidence of oncology nurses toward chemotherapyinduced peripheral neuropathy (CIPN) among patients with cancer in Jordan.

Methods: Descriptive, and cross sectional study was conducted on a convenience sample of 148 Jordanian registered oncology nurses recruited from a Specialized Oncology Center in Jordan. Data collection took place in June, 2015. As a data collection tool, the assessment of oncology nurses' knowledge and practice-revised questionnaire was used.

Results: The mean CIPN knowledge score of 8.98/16 $(\mathrm{SD}=1.9)$ indicated that there were knowledge deficits regarding CIPN among Jordanian oncology nurses. Only $58.8 \%$ of the subjects indicated that CIPN assessment is necessary for their oncology practices, but the majority rated their CIPN assessment skills as inadequate. Neurologic physical assessment usually is not included in practices assessment. In addition, $57.1 \%$ believed that CIPN was a significant problem for patients and their families.

Conclusion: There is a significant requirement to enhance the neurological assessment skills of oncology nurses and an efficient approach to CIPN assessment is demanded. Creating guidelines to manage and assess CIPN, and further investigations in different health institutions to generalize the results across Jordan are highly needed.

Saudi Med J 2018; Vol. 39 (11): 1158-1163 doi: 10.15537/smj.2018.11.23303

\footnotetext{
Chemotherapy-induced peripheral neuropathy is a common health complication that involves patients with cancer receiving neurotoxic chemotherapy. ${ }^{1}$ The incidence of CIPN among patients with cancer is estimated approximately $38 \%$ depending on the duration of exposure and the use of common types of chemotherapy involving platinum-based compounds, plant alkaloids, taxanes, and bortezomib. ${ }^{2}$ Chemotherapy-induced peripheral neuropathy involves
}

sensory symptoms such as numbness, tingling, pain, burning, balance problems, and cold sensation. ${ }^{3}$ The CIPN is one of the symptoms that can negatively affect the quality of life (QoL), treatment plan, and safety of patients. Chemotherapy-induced peripheral neuropathy affects daily activities, physical function, safety of patients with cancer, and ultimately causes difficulty to perform daily tasks. ${ }^{4}$ Neuropathic pain (NP) is a consequence of CIPN; however, oncology nurses are assessing and managing the general pain in patients with cancer without differentiating between nociceptive and neuropathic pain reflected poorly in the management of CIPN and consequently affecting patients' QoL negatively. ${ }^{5}$

Nurses are the largest group of health care providers in the position of identifying CIPN; therefore, knowledgeable oncology nurses can improve patients' QoL and improve patients' outcomes. ${ }^{6}$ Oncology nurses' knowledge about CIPN is limited; one of the causes is that nervous system and neurological assessments are not among the oncology nurse's educational targets, and until now there are no standard guidelines to assess and manage CIPN.' Chemotherapy-induced peripheral neuropathy is still one of the many challenges oncology nurses face, due to knowledge deficits and lack of diagnosis and management guidelines. Informing nurses about CIPN can affect the treatment and QoL of the patients. ${ }^{8}$ In fact, early assessments of CIPN in patients with cancer can prevent patient's injury and minimize complications by providing early intervention and management. ${ }^{9}$

In Jordan, however, only a few research studies were found concerning oncology nurses' knowledge and practices toward cancer pain in general, and even fewer research studies were conducted to assess oncology nurses' knowledge and practices toward CIPN. ${ }^{66}$ Therefore, the current study aimed at assessing knowledge, attitude, and practice (KAP) of oncology nurses toward CIPN among patients with cancer in Jordan. Accordingly, CIPN: assessment of oncology nurses' knowledge and practice-revised questionnaire was used. ${ }^{7}$

Benner theory of how nurses acquire their knowledge and expertise was used in the current study, specifically her theoretical idea about using descriptive accounts of clinical practice to examine the knowledge embedded in

Disclosure. Authors have no conflict of interests, and the work was not supported or funded by any drug company. 
nursing practice. Benner believed that good experience and education level lead to more proficiency in nurses, improve their decision making abilities and skills, and reflect the level and depth of caring. ${ }^{7}$ Nurses` level of knowledge and experience can affect their clinical skills and practices in dealing with CIPN, which in turn influences QoL and treatment outcomes of patients with cancer.

Worldwide, oncology nurses still lack the knowledge, assessment skills, screening, and management of CIPN. ${ }^{4-7,10}$ Therefore, to gain the needed power, oncology nurses need to be knowledgeable about all the aspects of CIPN, from the diagnosis to the management approaches; also patients' education and experience about CIPN can enhance their treatment plan and ultimately improve their QoL. ${ }^{6}$ According to a cross sectional, exploratory study conducted in the United States of America, results indicated nurses' knowledge deficit toward assessment of CIPN, inadequate training, and lack of expertise and confidence to evaluate neurologic physics. Nurses pointed out the need for CIPN assessment guidelines to improve the quality, safety, and working conditions in the oncology setting. ${ }^{7}$ Similarly, another study was conducted in 2014 to investigate the KAP level of nurses toward CIPN in the United States of America; sample included 408 oncology nurses, the findings indicated nurses' knowledge deficit regarding neurotoxicity, management, assessment skills, and measurement tools of CIPN.5

Visovsky et al, ${ }^{11}$ evaluated nurses' knowledge, practices, confidence, and skills in CIPN by a selfassessment survey, and a telephone conference was held at the end of the study with all participating nurses to discuss the barriers and facilitators in the implementation of CIPN assessments. Result showed no significant changes in the nurses' knowledge of CIPN from pre-test to post-test, and it can be attributed to poor internal consistency in nurses' knowledge and practices, but there were significant changes in nurses' confidence and skills to assess CIPN. Oppositely, there were no significant differences in nurses' knowledge and practices between certified and non-certified oncology nurses, or based on nursing degree.

Nurses play a critical role in caring for patients with CIPN. Nurses' information about CIPN assessment at baseline and before each cycle of chemotherapy is important. Oncology nurses should educate the patients about signs of CIPN for better management and enhancement of QoL; also, nurses' knowledge about chemotherapy and adjuvant medications can reduce the severity of CIPN. ${ }^{9}$

Nurses` knowledge about evidence-based practice in assessment, prevention, and management of CIPN are important, and at the same time, nurses` role in researches and measuring the effectiveness of interventions and management help to improve QoL in patients with CIPN. ${ }^{11}$ Nurses should also be knowledgeable about pharmacological and non-pharmacological approaches from evidence-based practices to better control, prevent, and minimize neurotoxicity outcomes caused by CIPN; and it was consistent with the conclusions that oncology nurses understanding of available pharmacological and non-pharmacological treatments can prevent and reduce CIPN outcomes. ${ }^{2}$

Nurses are advocates of patients, and to gain power in this side, nurses should be informed, and educate patients about CIPN, and need to have ongoing assessment and communication with them; therefore, patients with CIPN experience nurses' and health care teams help in the treatment plan, to choose the best treatment intervention. ${ }^{4}$

Methods. A descriptive, quantitative, and cross sectional study was conducted to assess the KAP level of oncology nurses toward CIPN. A total of 148 Jordanian oncology registered nurses at least with a bachelor degree, and certified as chemotherapy administration nurses were recruited conveniently from the specialized oncology center in Jordan. Worth noting that requirements for being a nurse in Jordan is to be graduated from a nursing degree program (locally at or outside countries) and have at least 1-2 years of experience are the only requirements to be employed as a nurse. However they do prefer the Bachelor degree holders. Unfortunately, there is no licensure or certification of specialty on the graduate level. Therefore, nurses with less than one year of employment in the center were excluded. The current study was ethically approved by the local center review board (IRB) in June 2015. The study objectives, as well as the inclusion criteria were given in a letter to the participants. The letter also had some instructions to complete the questionnaire that was implied as consent, and indicating that the participation was confidential and voluntary. The participants were also assured about the confidentiality of their information. The questionnaires were anonymous and the study had no instrument to identify the respondents. A self-reporting questionnaire was used to collect data. About 20-30 minutes was required to fill out the questionnaire; the required data were gathered within 10 days. The collected data were transferred to Microsoft Office Excel 2007 and analyzed with SPSS version 24.0 (IBM Corp., Armonk, NY, USA). Missing data were considered as missing value codes; missing data for the knowledge items were considered incorrect. 
Chemotherapy-induced peripheral neuropathy: assessment of oncology nurses' knowledge and practicerevised instrument was used in the current study. This instrument assesses the KAP level of oncology nurses toward CIPN; the questionnaire consisted of 16 knowledge items and the scores range $0-16 ; 16$ practice and behavior items, scored based on a 4-option Likert scale ranged 0-3 (never, occasionally, frequently, and always) or (not at all confident, somewhat confident, confident, and very confident); one item about assessment skills, 3 items about previous instruction, 4 items about nurses' perception, and 9 demographical characteristics items. ${ }^{7}$ The reliability of the questionnaire was confirmed by Cronbach alpha (0.84). Also, the questionnaire was validated by $3 \mathrm{PhD}$ holder expert nurses in CIPN, and 2 medical oncologists. The questionnaire content validity index was $(0.95){ }^{7}$

Results. The demographic characteristics of the nurses are shown in Table 1. The convenience sample for the current study included 148 oncology nurses constituted $69(46.6 \%)$ females. The mean age of nurses was (27.23) years, ranged 23-46. In terms of the educational level, most of the respondents had Bachelor (91.9\%). The nursing experience ranged 1-26 years, and specific experience in oncology nursing ranged $(<2-17)$ years.

To measure CIPN knowledge, the true responses of 16 items were expressed as percentage. Items evaluating knowledge included signs and symptoms of CIPN, CIPN-inducing chemotherapy agents, and patients information including functional impairment, subjective symptoms, and neurological changes attributed to CIPN.

The mean knowledge score of $8.98 \pm 1.9$ (maximum score $=16)$. The areas of inadequate knowledge were essential as the first step to assess CIPN (33\%), CINP sensory symptoms mainly proximal to distal pattern progression $(35 \%)$, any critical signs in the assessment of CIPN (36\%), non-pharmacological strategies to manage CIPN symptoms (42\%), and patients' reports of peripheral neuropathy (PN) symptoms (49\%).

To assess the practice of participants, a self-reporting system including patterns of screening, skills of assessment, documentation, and teaching patients about CIPN were used (Figure 1).

Practice and assessment frequency of CIPN were scored based on a 4-option Likert scale $(1=$ never, $2=$ occasionally, $3=$ frequently, and $4=$ always). Screening patterns before injection of the first dose of neurotoxic chemotherapy, PN was always or mostly screened by 48 (32.50\%) nurses. A percent of 36.5 noted they frequently assessed patients before each infusion. Assessment skills
Table 1 - Demographic characteristics of oncology nurses ( $N=148)$.

\begin{tabular}{lccc}
\hline Characteristics & Minimum & Maximum & Mean \pm SD \\
\hline Age & 23 & 46 & $27.23 \pm 3.567$ \\
Years in nursing & 1 & 26 & $4.89 \pm 3.544$ \\
Years in oncology & 0 & 17 & $3.86 \pm 2.649$ \\
nursing & & & \\
Valid N (list wise) & 148 & & \\
\hline Characteristics & Percent & Valid & Cumulative \\
& & percent & percent \\
\hline Gender & & & \\
Male (n=79) & 53.4 & 53.4 & 53.4 \\
Female (n=69) & 46.6 & 46.6 & 100.0 \\
$\quad$ Total (n=148) & 100.0 & 100.0 & \\
Education & & & \\
Diploma (n=2) & 1.4 & 1.4 & 1.4 \\
Bachelor (n=136) & 91.9 & 91.9 & 93.2 \\
Masters (n=10) & 6.8 & 6.8 & 100.0 \\
Total (n=148) & 100.0 & 100.0 & \\
\hline \multicolumn{4}{l}{} \\
\hline
\end{tabular}

of fine motor skills were always or mostly assessed by $54(36.5 \%)$ nurses. Only 34 (23.0\%) nurses always or mostly assessed the deep tendon reflexes. Also, 37 $(25.0 \%)$ nurses always or mostly measured muscle strength. Risk factors associated with PN were always or frequently evaluated by $42(28.4 \%)$ nurses. Fine motor function (gait assessment) was all the times or repeatedly evaluated by $63(42.6 \%)$ nurses. $45(30.4 \%)$ oncology nurses always or frequently attempted CIPN evaluation before neurotoxic agent infusion. Chemotherapyinduced peripheral neuropathy was always or frequently checked by 49 (33.1\%) nurses. Chemotherapy-induced peripheral neuropathy was always or frequently assessed by $48(32.4 \%)$ nurses. Regarding documentation only $32.4 \%$ documented CIPN assessment data. While, patient teaching related to CIPN adapting strategies to CIPN functional impairment was always or frequently taught to patients by $41(27.7 \%)$ oncology nurses. Safety precautions used to avoid injury were repeatedly taught to the patients with CIPN by 97 (65.5\%) oncology nurses.

Self-confidence was assessed in the subjects $(n=148)$ by NP evaluation including deep tendon reflexes, tuning fork vibration sensation, sharp vs. dull sensation, the Romberg test, and manual muscle strength test (Figure 2); 113 (76.4\%) nurses reported low confidence to assess deep tendon reflexes, $105(71.0 \%)$ use a tuning fork to assess vibration sensation, and $111(75.0 \%)$ perform the Romberg test. Only, 44 (29.7\%) nurses reported good confidence to perform the sharp vs. dull sensation assessment skills, and $52(35.1 \%)$ nurses were confident to assess manual muscle strength. 
Only $58.8 \%$ of the subjects believed that CIPN assessment was necessary for their oncology practices. Although $57.1 \%$ of the nurses noted CIPN as a remarkable health problem of patients, only $40.8 \%$ reported receiving instruction in this regard. A large amount of knowledge and practice deficit was reported by the nurses without any information about CIPN.

Reasons remarked for not routinely attempting CIPN evaluations were inadequate competence, CIPN not included in the assessment sheet, CIPN assessment perceived as the responsibility of the physician, time constraints, no standard guidelines to assess CIPN, and receiving no prior education related to CIPN. Nurses welcomed more education in NP assessment skills to more precisely evaluate CIPN.

Education and experience level of participants correlation with the knowledge about CIPN ( $n=148)$.

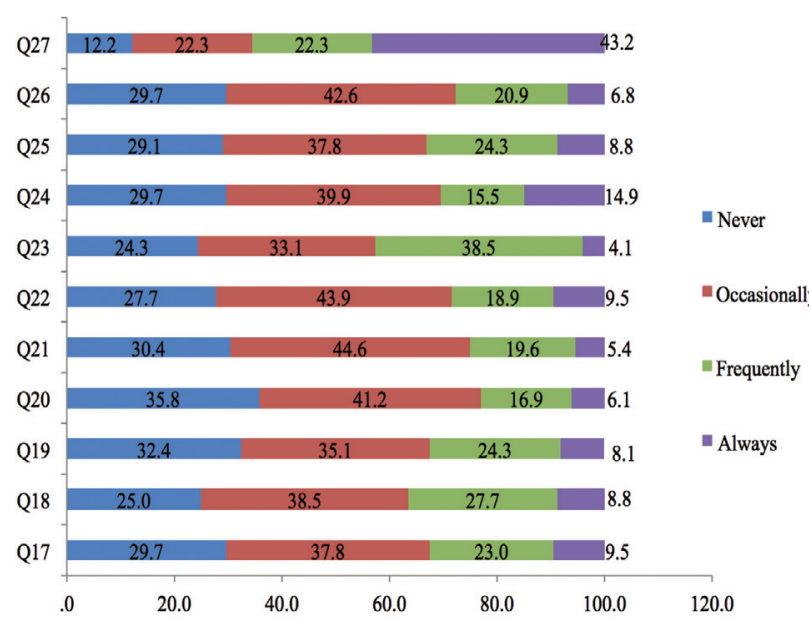

Figure 1 - Practice/Behaviors. Q17) How often do you screen patients for baseline presence of peripheral neuropathy prior to initiating the first dose of chemotherapy? Q18) How often do you assess patient's ability to perform fine motor skills (namely, button clothes, use of zippers) if they are receiving neurotoxic chemotherapeutic agents? Q19) How often do you document CIPN assessment data if the patient is receiving chemotherapy associated with CIPN? Q20) How often do you assess deep tendon reflexes on patients receiving neurotoxic chemotherapy drugs? Q21) How often do you assess muscle strength in patients receiving neurotoxic chemotherapy? Q22) How often do you assess patients for the presence of other risk factors associated with peripheral neuropathy? Q23) How often do you perform objective motor function assessment skills (namely, muscle strength, gait assessment)? Q24) How often do you perform nursing assessment of CIPN prior to each infusion of neurotoxic chemotherapy? Q25) How often do you attempt to elicit patient symptoms related to chemotherapy-induced peripheral neuropathy? Q26) How often do you teach patients strategies for adapting to functional impairments secondary to CIPN? Q27) How often do you educate patients about safety precautions used to avoid injuries associated with CIPN (namely, thermal injury, falls)?

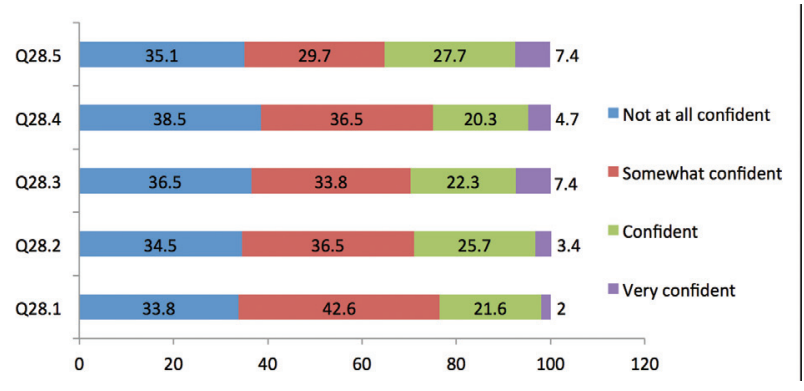

Figure 2 - Level of confidence. Q28) Check your level of confidence in performing each of the following physical assessment skills: Q28.1) Deep tendon reflexes. Q28.2) Tuning fork vibration sensation. Q28.3) Sharp vs. dull sensation. Q28.4) Romberg test. Q28.5) Manual muscle strength testing.

Based on Pearson correlation, the association of generic nursing education with CIPN knowledge was statistically insignificant; the same relationship was also observed between the academic degree and CIPN knowledge. However; relatively, a significant relationship between years of experience in nursing and CIPN knowledge $(\mathrm{r}=0.926, p=0.008)$; similarly, observed relationship between specific experience in oncology nursing, and CIPN knowledge $(\mathrm{r}=0.472, p=0.060)$.

Discussion. Chemotherapy-induced peripheral neuropathy is one of the major depleting consequences of chemotherapy. Nurses should have a major role in assessment and management of CIPN, to counteract the negative impact on the patient's QoL. Due to scarcity of research assessing nurses' knowledge and attitudes toward CIPN it is difficult to determine if nurses are adequately prepared to conduct this role. The current study aimed at assessing the KAP level of oncology nurses toward CIPN. So, this study expanded current knowledge regarding this matter; therefore the study findings will guide future interventional efforts to translate evidence based knowledge into clinical practice.

Nursing specialists and the clinical educators holding undergraduate degrees constituted the majority of participants. The sample comprised of oncology practice settings such as inpatient and outpatient clinic nurses, as well as infusion nurses. The mean score of knowledge $(8.98 \pm 1.992)$ indicated inadequate knowledge toward CIPN. Areas of inadequate knowledge were non-pharmacological approaches to control CIPN symptoms, autonomic neuropathy appearing as orthostatic hypotension, and CIPN sensations terminology. Low mean score of knowledge could be explained from the perspective that many 
oncology nurses work in subspecialty areas caring for very specific patient populations (namely, colon, breast) and are not as familiar with treatments used to treat all cancer types. Another possible explanation, nurses may have inaccurately responses based on their beliefs regarding what should be carried out, and not based on their actual practice behaviors.

It is worth noting that in most cases, studies were not directly comparable because of different measurements, and questionnaires used. However, this finding is largely consistent with other studies that reported inadequate knowledge toward CIPN. ${ }^{5,7}$ However, the mean score of knowledge $(8.98 / 16)$ is significantly lower than what was reported in the study conducted in United States of America (12.6/16).7 This inconsistency might be related to the longer exposure to pain education, and specialty certification which can positively impact nurses' knowledge and higher scores in United States of America. Also, in the study by Smith et al, ${ }^{5}$ the majority of the sample was nurses with master's degrees and above.

Nurses have adequate knowledge about CIPN risk factors, but not routinely attempted for screening, represented a knowledge-practice gap. The findings of the current study indicated that nurses had no confidence regarding physical assessment skills to perform deep tendon reflexes, tuning-fork vibration, and the Romberg testing and only somewhat confident to evaluate muscle strength. Indicating an absence of best practices for knowledge integrated into practice. The oncology nurses noted the priority of CIPN assessment in their clinical practices; however, $75 \%$ believed no remarkable role for CIPN assessment skills. There was a remarkable knowledge inadequacy in nurses without any history of CIPN assessment instructions. Indicating that the foundational nursing knowledge required for CIPN assessment was inadequate. This may be due to that comprehensive neurologic examination may not be part of a nurse education at the graduate level.

Poor knowledge of assessment skills, time limits, and considering the documentation form as an inappropriate form to utilize the process of assessment were among the reasons the nurses noted not to routinely perform CIPN assessments. The current findings like other published reports suggesting such barriers to CIPN assessment. ${ }^{5,7,11}$

Self-confidence of nurses toward CIPN assessment is shown in Figure 1; $76.4 \%$ of the nurses were highly confident to perform sharp vs. dull sensation as well as manual muscle strength testing; however, they felt poor confidence to assess deep tendon reflexes, test tuning fork vibration sensation, and the Romberg testing. Simple assessment tools are required for sharp versus dull sensation and manual muscle strength testing. Higher levels of assessment knowledge to perform tuning fork vibration sensation, deep tendon reflexes, and the Romberg testing are required by nurses with poor confidence. Oncology nurses may be never trained for such skills. A neurological assessment skill is of the duties of clinicians. The level of confidence was not reported by Binner. ${ }^{7}$

Nurses were not confident in performing NP assessment skills based on the level of confidence and frequency of practice. All respondents believed that CIPN assessment is necessary for oncology nurse in charge of neurotoxic chemotherapy administration; $76.45 \%$ of nurses believed they had fair or poor skills to assess CIPN. Xue et al, ${ }^{12}$ also indicated that $80 \%$ of nurses reported their fair or poor education and training about cancer pain. Lack of confidence in CINP assessment skills may be a factor in the knowledgepractice gap.

There was a poor and significant relationship between the academic degree and CIPN knowledge; however, the relationship between nursing experience and CIPN knowledge was insignificant. The nurses had various educational levels; mostly undergraduates followed by MSc degree and $\mathrm{PhD}$. Based on the findings, there was a positive association between higher education and knowledge in CINP and the ability to use such knowledge in clinical settings. The relationship was not reported by Binner. ${ }^{7}$

Recommendations and implications. The current study helps the oncology nurses to anticipate evidencebased educational programs covering prevention and treatment of CIPN and the use of complementary therapies required to be integrated into oncology nurse orientation and to be updated frequently; in practice, they can help nurses to have standard guidelines in the assessment and management of CIPN. For the education part, the study highlighted the need of oncology nurses for solid information, and educational programs to successfully develop their practice and behaviors in dealing with patients with CIPN. For researchers, a retrospective study on the patients 'files previously complained about CIPN, and how the nurses and physicians assessed and managed them is helpful to measure the outcomes. The health care system in Jordan is considered a decentralized system that consists of governmental, military, private, and non-profit organizations, which need further studies in each system to fill the gap in CIPN management and assessment to create a national guideline to cover all health system institutions. 
Limitations. The current study had limitations in terms of its ability to be generalized to the total number of oncology nurses in Jordan, since data were collected from a single oncology site in Jordan; another potential bias was that responses may be indicated on how nurses believe they should practice rather than actual things or the correct practices.

In conclusion, The KAP of oncology nurses toward CIPN in patients with cancer is studied in a number of studies so far. The current study demonstrated the state of oncology nurses' practices and the results supported the need to train the oncology nurses about neurological assessment skills. To develop a useful approach for CIPN assessment, time limits of the outpatient setting and the proficiency of nurses in CIPN evaluation should be considered. The roles and responsibilities of nurses to assess CIPN should be interpreted to overcome the ambiguity.

Acknowledgment. The authors would like to thank all nurses that participated in the study, and they would like to thank Khawlah Ammar, Dalia Al-Rimawi, and Hadeel Abdelkhaleq for data analysis and statistical support; and Madelaine Binner, for her generosity in sharing the measurement tool. Finally, a special thanks to NedMedic for English language editing.

Received 9th July 2018. Accepted 10th September 2018.

From the Department of Adult Health Nursing (Al-Atiyyat), Faculty of Nursing, Hashemite University, and from the Training Center (Banifawaz), King Hussein Cancer Center, Zarqa, Jordan.

Address correspondence and reprints request to: Dr. Nijmeh M. Al-Atiyyat, Department of Adult Health Nursing, Faculty of Nursing, Hashemite University, Zarqa, Jordan.E-mail:nijmeh@hu.edu.jo

ORCID ID: orcid.org/0000-0002-6519-5481

\section{References}

1. Gornstein EL, Schwarz TL. Neurotoxic mechanisms of paclitaxel are local to the distal axon and independent of transport defects. Exp Neurol 2017; 288: 153-166.
2. Kerckhove N, Collin A, Condé S, Chaleteix C, Pezet D, Balayssac D. Long-Term Effects, Pathophysiological Mechanisms, and Risk Factors of Chemotherapy-Induced Peripheral Neuropathies: A Comprehensive Literature Review. Front Pharmacol 2017; 8: 86.

3. Hershman DL, Lacchetti C, Loprinzi CL. Prevention and Management of Chemotherapy-Induced Peripheral Neuropathy in Survivors of Adult Cancers: American Society of Clinical Oncology Clinical Practice Guideline Summary. J Oncol Pract 2014; 10: e421-e424.

4. Tofthagen C, Visovsky CM, Hopgood R. Chemotherapyinduced peripheral neuropathy: an algorithm to guide nursing management. Clin J Oncol Nurs 2013; 17: 138-144.

5. Smith EM, Campbell G, Tofthagen C, Kottschade L, Collins ML, Warton C, et al. Nursing knowledge, practice patterns, and learning preferences regarding chemotherapy-induced peripheral neuropathy. Oncol Nurs Forum 2014; 41: 669-679.

6. Al-Atiyyat N, Obaid A. Management of peripheral neuropathy induced by chemotherapy in adults with cancer: a review. Int J Palliat Nurs 2017; 23: 13-17.

7. Binner M, Ross D, Browner I. Chemotherapy-induced peripheral neuropathy: assessment of oncology nurses' knowledge and practice. Oncol Nurs Forum 2011; 38: 448-454.

8. Schönsteiner SS, Bauder Mißbach H, Benner A, Mack S, Hamel $\mathrm{T}$, Orth $\mathrm{M}$, et al. A randomized exploratory phase 2 study in patients with chemotherapy-related peripheral neuropathy evaluating whole-body vibration training as adjunct to an integrated program including massage, passive mobilization and physical exercises. Exp Hematol Oncol 2017; 6: 5.

9. Taverner T. Neuropathic pain: an overview. British Journal of Neuroscience Nursing 2014; 10: 116-123.

10. Haryani H, Fetzer SJ, Wu CL, Hsu YY. Chemotherapy-Induced Peripheral Neuropathy Assessment Tools: A Systematic Review. Oncol Nurs Forum 2017; 44: E111-E123.

11. Visovsky C, Haas M, Faiman B, Kurtin S, Shaftic AM, Lyden E, et al. Nurse self-evaluation of assessment of chemotherapyinduced peripheral neuropathy in patients with cancer. $J A d v$ Pract Oncol 2012; 3: 319-325.

12. Xue Y, Schulman-Green D, Czaplinski C, Harris D, McCorkle R. Pain attitudes and knowledge among RNs, pharmacists, and physicians on an inpatient oncology service. Clin J Oncol Nurs 2007; 11: 687-695. 